\title{
Association of Burnout with Emotional Coping Strategies, Friendship, and Institutional Support Among Internal Medicine Physicians
}

\author{
Benjamin R. Doolittle ${ }^{1}$ (D)
}

Published online: 15 May 2020

(c) Springer Science+Business Media, LLC, part of Springer Nature 2020

\begin{abstract}
Physician burnout influences physician mental health, staff stress, safety events, and patient outcomes. The association of burnout with compassion satisfaction, secondary stress, emotional coping strategies and many psychosocial variables, such as institutional support, friendship, and spirituality, have not been well studied. A convenience sample of internal medicine physicians was emailed a survey using validated instruments to explore these associations. The response rate was $337 / 1021$ (33\%), with a burnout prevalence of 175/337 (52\%). Grit, acceptance, active coping, positive reframing, and strategy planning were associated with lower burnout domains and greater compassion satisfaction. Certain emotional coping strategies such as denial, disengagement, self-blame, substance abuse, and venting were associated with greater burnout and lower compassion satisfaction. Greater institutional support was associated with lower burnout $(r=-.35, p<.001)$, secondary stress $(r=-.14, p<.05)$, and compassion satisfaction $(r=.28, p<.0001)$. Friendship was associated with lower burnout $(r=-.25$, $p<.0001)$ and greater compassion satisfaction $(r=.28, p<.0001)$. This study suggests that amelioration of burnout requires both intrinsic strategies that emphasize physician coping skills as well as extrinsic strategies that address institutional support.
\end{abstract}

Keywords Burnout $\cdot$ Physician $\cdot$ Coping $\cdot$ Emotional health $\cdot$ Compassion fatigue $\cdot$ Secondary stress

Physician burnout is associated with worse patient care, increased medical errors, substandard professionalism, and poor mental health (Williams, Manwell, Konrad, \& Linzer, 2007). To complement the triple aim of improving health, ameliorating the patient experience, and reducing costs, the importance of improving physician wellness has been heralded as "the fourth aim" (Bodenheimer \& Sinsky, 2014). Burnout is defined as a constellation of three domains: emotional exhaustion, where the provider is depleted of emotional, spiritual, and physical energy, depersonalization, characterized by cynicism and objectification of the other, and reduced personal accomplishment, marked by feelings of low job satisfaction (Maslach, Jackson, \& Leiter, 1996) $44 \%$ of the physician population meets these criteria (Shanafelt et al., 2019). Demographic variables that correlate with burnout have been well described. Physicians experiencing burnout tend to be earlier in their career, as they are

Benjamin R. Doolittle

Benjamin.doolittle@yale.edu

1 Departments of Internal Medicine and Pediatrics, Yale University School of Medicine, 333 Cedar Street 1074LMP, PO BOX 8033, New Haven, CT 06520, USA establishing themselves in their first jobs, contending with debt, and starting a family (Chopra, Sotile, \& Sotile, 2004). Reduced burnout is associated with male physicians, those who are married, and having children (Chopra et al., 2004). Other work-related variables are associated with increased burnout: longer work hours, increased patient care responsibility, demands imposed by working with electronic medical records (EMRs), and lack of continuing education (Chopra et al., 2004). Even as EMRs have evolved, physician burnout is associated with dissatisfaction with their clerical burdens and interference with direct patient care (Shanafelt et al., 2016).

Physician demographics are only one aspect of burnout. Several intrinsic and extrinsic qualities are associated with less burnout. Emotional strengths, such as resilience and optimism, can be an important coping strategies (Doolittle, Windish, \& Seelig, 2013). Also, institutional support, as by defined by physicians' perception that they are mentored and encouraged by departmental leaders, is associated with lower burnout (Yost, Eshelman, Raoufi, \& Abouljoud, 2005). Social support and friendship have been shown to correlate with improved quality of life and burnout in at least two small cohort studies among Canadian rural practitioners 
and Serbian physicians (Lavanchy et al., 2004; Vicentic et al., 2013). Religion, from personal spirituality to organized religious practice, has also been shown to correlate with improved mental health, especially depression, anxiety disorders, and suicidality (Bonelli and Koenig, 2013). Friendship, social support, religion, and institutional support have not been widely studied among internal medicine physicians. Further, resilience, the quality of persevering despite difficulties, has emerged as a recent construct in the burnout conversation but has shown only a weak correlation with reduced burnout among physicians (Halliday, Walker, Vig, Hines, \& Brecknell, 2017).

In addition, there are other constructs that inform burnout, such as compassion satisfaction and secondary stress (Stamm, 2005). Compassion satisfaction is the sense of achievement, purpose, and joy generated from pursuing emotionally demanding work (Stamm, 2005). In contrast, secondary stress, also known as compassion fatigue, highlights the acquired stress that practitioners experience while attending to the intense emotional needs of their clients. Secondary stress is specifically related to caregiver strain from treating trauma victims and mirrors the symptoms of post-traumatic stress disorder, with such symptoms as hyperarousal, numbness, anxiety, and avoidance. While similar, burnout is a different construct that investigates job-related exhaustion, disengagement, and low satisfaction. Although tightly correlated, secondary stress and burnout evolved from different research questions (El-bar et al., 2013). Practitioners can be energized by their role as healers or, in turn, internalize the stress and suffering experienced by patients. These concepts merit further exploration in the context of physician burnout and well-being.

Given the importance of physician burnout in the literature, this study investigates the association of burnout among internal medicine physicians with compassion satisfaction, secondary stress, and emotional coping strategies, such as resilience. Further, we investigate the correlation of social support, such as institutional culture and friendship, that may correlate with the burnout syndrome.

\section{Methods}

\section{Study Design and Sample}

This study cohort was a convenience sample of attending internal medicine physicians who were members of the American College of Physicians, Connecticut Chapter, and the American College of Physicians from the Hudson Valley Region of upstate New York. They were asked to complete an anonymous, confidential, and voluntary survey via a weblink on three separate occasions in May 2015.

\section{Survey Instrument}

All variables were evaluated using validated instruments. The survey contained demographic data and five validated instruments: the Professional Quality of Life Scale (ProQol) (Stamm, 2005), the COPE Inventory (Carver, Scheier, \& Weintraub, 1989), the Grit Scale (Duckworth, Peterson, Matthews, \& Kelly, 2007), the Duke University Religion Index (DUREL) (Koenig \& Bussing, 2010), and the Rapid Assessment of Physical Activity (RAPA) (Topolski, LoGerfo, Patrick, Williams, Walwick, \& Patrick, 2006). Demographic information included age, gender, income, marital status, years in practice, children, and practice setting.

The ProQol has been used in more than 200 studies to evaluate three domains: burnout, secondary stress (also known as compassion fatigue), and compassion satisfaction (Stamm, 2005). The ProQol includes 30 items scored on a five-point scale $(1=$ never, $2=$ rarely, $3=$ sometimes, $4=$ often, $5=$ very often). Each domain can be treated as a continuous variable, while standardized cut-offs provide guidance about high levels of each domain. A score greater than 25 in the burnout domain defines "high burnout" (Stamm, 2005).

The COPE inventory comprises 15 four-item scales that assess a variety of coping strategies, including adaptive and maladaptive strategies (Carver, Scheier, \& Weintraub, 1989). Participants indicate how frequently they use each coping strategy on a four-point scale anchored by "usually do not do this at all" and "usually do this a lot." The COPE inventory was specifically designed to evaluate an individual's responses to a stressful environment. Based on both a theoretical and empirical model, the COPE inventory incorporates both "problem-focused" and "emotionfocused" coping strategies and addresses the deficiencies of prior instruments.

The Grit Scale measures resilience using 12 items on a 5-point Likert scale ("very much like me," "mostly like me," "somewhat like me," "not much like me," "not like me at all") (Duckworth et al., 2007). The Grit Scale is used to address resilience defined as perseverance and passion for long-term goals.

Religious involvement was evaluated using the Duke University Religion Index (DUREL) (Koenig \& Bussing, 2010). The DUREL is a 5-item instrument that explores issues of both religious practice and intrinsic belief on a six-point Likert scale. Physicians were also asked subjectively if they considered themselves religious (yes/no) or spiritual (yes/no). Exercise was investigated the Rapid Assessment of Physical Activity (RAPA), a nine-item instrument to assess for physical exercise, strength and flexibility training, that is tailored toward middle age and 
older adults (Topolski et al., 2006). There were no validated instruments for institutional support and friendship that were applicable for this study. These two domains were explored on a 1-10 Likert scale, "How well does your institution support you?" and "How well do your friends support you?"

\section{Statistical Analyses}

We used JMP version 4.04 (SAS, Cary, NC, USA) for all statistical analyses. We calculated the scores of the burnout inventory, COPE, Grit Scale, and DUREL instruments. We used the Student's t test to compare the means of ProQol categories with demographic variables and nominal variables. Continuous variables (age, years in practice, and income) were correlated with the Spearman rank coefficient. Multivariate regression modeling was also employed to ascertain the impact of these variables upon the ProQol domains. Given that multiple variables are correlated with the three ProQol domains, a Bonferroni correction was made to make the significance level of $p<.004$ (alpha $=0.05,13$ observations, corrected $p=0.5 / 13=0.004)$ (Sedgwick, 2012).

\section{Results}

From a combined mailing list of 1,021 physicians, 337 physicians responded, resulting in a $33 \%$ response rate. Demographic composition of the responding sample showed that most respondents were male (56\%), with an average age of 52 years of age $( \pm 12)$ and 20 years $( \pm 11)$ in practice. (Table 1).

Among the respondents, 175 (52\%) met criteria for burnout. $206(61 \%)$ considered themselves spiritual. 128 (38\%) considered themselves religious. 239 (71\%) self-reported that they exercised regularly. $280(83 \%)$ reported that they had a close friend. There was no difference between regions in any variable measured. (Table 2).

Older age correlated with lower burnout scores, and secondary stress scores, and with higher compassion satisfaction scores. Similarly, years in practice was inversely correlated with burnout, and secondary stress, and positively correlated with compassion satisfaction. Women were more likely to have higher levels of burnout $(p<.05)$ and secondary stress $(p<.02)$. Gender was not correlated with compassion satisfaction. Single physicians had higher burn out scores and lower compassion satisfaction scores than married, divorced or widowed physicians. Practice setting (inpatient, outpatient, or both) was not associated with burnout, secondary stress, or compassion satisfaction (Table 3 ).

Exercise ( 3 times per week for $20 \mathrm{~min}$ ) was associated with lower burnout $(p<.05)$, secondary stress $(p<.05)$, and greater compassion satisfaction $(p<.05)$ (Table 3$)$. Neither
Table 1 Demographic characteristics $(n=337)$

\begin{tabular}{lll}
\hline Demographic & Respondent sample & $\%$ \\
\hline Age (years) (sd) & $52(+/-12)$ & \\
Gender (men) & & 56 \\
Male & 189 & 44 \\
Female & 148 & \\
Years in practice & $20(+/-11)$ & \\
Average salary (dollars) (sd) & $219,000(+/-109,000)$ & 79 \\
Children & & 21 \\
Yes & 266 & \\
No & 71 & 79 \\
Marital status & & 11 \\
Married & 266 & 9 \\
Single & 37 & 1 \\
Divorced & 31 & \\
Widowed & 3 & 19 \\
Practice setting & & 48 \\
Inpatient & 65 & 33 \\
Outpatient & 162 & \\
Both & 110 & \\
\hline
\end{tabular}

Table 2 Prevalence of burnout, religiosity, and exercise variables $(N=337)$

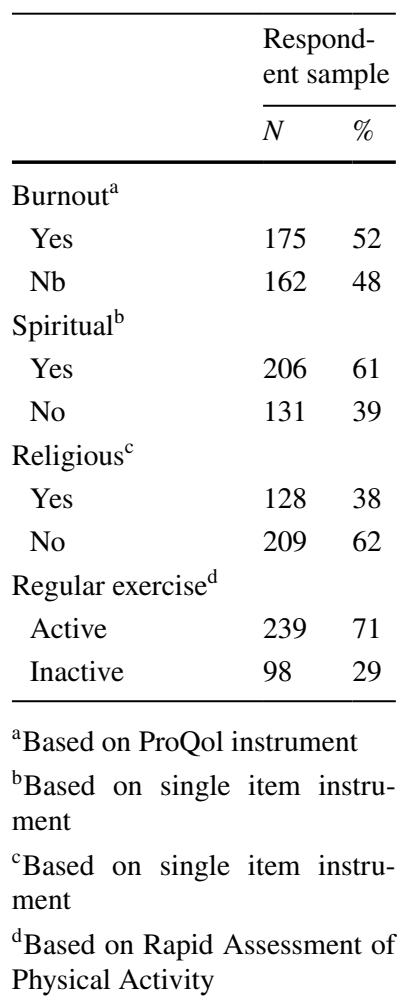

self-identifying as being religious or spiritual, nor the components of the DUREL—organized involvement, personal religious practice (e.g., prayer) and religiosity - were correlated with any ProQol domain of burnout, secondary stress, or compassion satisfaction (Table 4). Also, salary did not correlate with these same ProQol domains. 
Table 3 Univariate analysis of demographic variables with burnout, secondary stress, and compassion satisfaction (Student's $t$ test)
Table 4 Burnout, secondary stress, compassion satisfaction correlated with coping variables, friendship, religion, and institutional support

\begin{tabular}{|c|c|c|c|c|c|c|}
\hline \multirow[t]{2}{*}{$\mathrm{s}$} & \multicolumn{2}{|l|}{ Burnout } & \multicolumn{2}{|c|}{ Secondary stress } & \multicolumn{2}{|c|}{ Compassion satisfaction } \\
\hline & Mean (sd) & $p$ value & Mean (sd) & $p$ value & Mean (sd) & $p$ value \\
\hline \multicolumn{7}{|l|}{ Gender } \\
\hline Female & $26.91(6.11)$ & $p<.05$ & $20.17(5.27)$ & $p<.02$ & $33.93(5.99)$ & NS \\
\hline Male & $25.27(6.55)$ & & $29.01(4.89)$ & & $34.57(6.55)$ & \\
\hline \multicolumn{7}{|c|}{ Marital status } \\
\hline Married & $25.69(6.44)$ & $p<.01$ & $19.35(4.79)$ & NS & $34.52(6.25)$ & $p<.001$ \\
\hline Single & $29.25(5.60)$ & & $20.97(6.29)$ & & $30.69(5.63)$ & \\
\hline Divorced & $25.22(6.30)$ & & $19.16(5.85)$ & & $35.71(6.19)$ & \\
\hline Widowed & $25.44(6.10)$ & & $19.32(4.81)$ & & $34.66(6.10)$ & \\
\hline \multicolumn{7}{|l|}{ Children } \\
\hline Yes & $25.49(6.43)$ & $p<.01$ & $19.23(4.90)$ & $p<.05$ & $34.81(6.23$ & NS \\
\hline No & $27.86(6.01)$ & & $20.60(5.62)$ & & $32.39(6.24)$ & \\
\hline \multicolumn{7}{|l|}{ Exercise } \\
\hline Active & $25.11(6.28)$ & $p<.05$ & $18.92(4.78)$ & NS & $35.11(5.82)$ & $p<.01$ \\
\hline Inactive & $28.04(6.20)$ & & $20.27(5.36)$ & & $33.27(6.74)$ & \\
\hline \multicolumn{7}{|c|}{ Practice setting } \\
\hline Inpatient & $26.65(5.86)$ & NS & $20.07(7.34)$ & NS & $30.80(6.24)$ & NS \\
\hline Outpatient & $25.60(6.29)$ & & $20.28(4.53)$ & & $33.86(6.61)$ & \\
\hline Both & $26.2(6.88)$ & & $19.29(5.16)$ & & $34.66(6.22)$ & \\
\hline
\end{tabular}

\begin{tabular}{llll}
\hline & Burnout & Secondary stress & $\begin{array}{c}\text { Compassion } \\
\text { satisfaction }\end{array}$ \\
\hline Positive coping strategies & & & $+0.22^{*}$ \\
Acceptance & $-0.19^{* *}$ & $-0.15^{*}$ & $+0.43^{* * *}$ \\
Active coping & $-0.43^{* *}$ & -0.16 & $+0.41^{* *}$ \\
Grit & $-0.29^{* *}$ & -0.23 & $+0.20^{* *}$ \\
Positive reframing & $-0.18^{*}$ & $\mathrm{NS}$ & $+0.25^{* *}$ \\
Strategy planning & $-0.21^{* *}$ & $\mathrm{NS}$ & \\
Negative coping strategies & & & $-0.22^{* *}$ \\
Denial & $+0.30^{* *}$ & $+0.34^{* *}$ & $-0.44^{* *}$ \\
Disengagement & $+0.58^{* *}$ & $+0.37^{* *}$ & $-0.26^{* *}$ \\
Self-blame & $+0.38^{* *}$ & $+0.34^{* *}$ & $\mathrm{NS}$ \\
Substance abuse & $+0.12^{*}$ & +0.17 & $\mathrm{NS}$ \\
Venting & $+0.12^{*}$ & +0.12 & $+0.28^{* *}$ \\
Social support & & & $+0.28^{* *}$ \\
Institutional support & $-0.35^{* *}$ & -0.14 & $\mathrm{NS}$ \\
Friend support & $-0.25^{* *}$ & -0.12 & $\mathrm{NS}$ \\
Religious (yes/no) + & $\mathrm{NS}$ & $\mathrm{NS}$ & +0.14 \\
Spiritual (yes/no) + & $\mathrm{NS}$ & $\mathrm{NS}$ & +0.23 \\
Organized religion (DUREL) & -0.12 & -0.21 & +0.20 \\
Private practices (DUREL) & -0.21 & -0.12 & -0.18 \\
Religiosity (DUREL) & -0.18 & &
\end{tabular}

Adjusted for multiple comparisons with Bonferroni correction, $p<.004$ is level of significance $N S$ not significant

$* p<.004 ; * * p<.0001$

+ Student's $t$ test performed for comparing nominal variables with means or ProQol domains. All other measures are Pearson correlation coefficient 
Table 4 describes the correlation coefficients of the ProQol domains with emotional coping strategies, institutional support, and friendship. Of note, higher levels of grit were associated with lower burnout, lower secondary stress, and greater compassion satisfaction. Higher levels of institutional support were also associated with lower burnout and secondary stress, and greater compassion satisfaction. Greater support from friends was correlated with lower burnout and greater compassion satisfaction. These associations remained significant even when controlling for demographic variables.

Several emotional coping strategies were associated with lower burnout and secondary stress and greater compassion satisfaction: acceptance, active coping (i.e., perseverance), positive reframing (i.e., optimism), and strategy planning (i.e., designing a course of action). Several emotional coping strategies were associated with greater burnout, secondary stress and lower compassion satisfaction: denial, disengagement, self-blame, substance abuse, and venting. Table 5 presents the correlations between burnout, secondary stress, and compassion satisfaction.

In multivariate modeling, grit, institutional support, friendship, exercise, and the COPE inventory accounted for $55 \%(p<.0001)$ for the variation of burnout, $43 \%(p<.001)$ for compassion satisfaction, and 33\% $(p<.0001)$ of secondary stress. Given the low response rate, post hoc power calculations were performed. Given the sample size of 337 , an alpha 0.05 , and an effect size of 0.2 , the study is adequately powered at $98 \%$.

\section{Qualitative Data}

Participants were invited to write in comments about major stressors. One respondent wrote, "I feel like a hamster on a wheel." Others cited a "lack of control," and "unrealistic expectations." Another participant wrote, "Being blamed for things over which I have no control, such as whether a woman has had a mammogram. I can write an order, but short of placing her breast on the plate, there is not much I can do to make sure it happens... I could go on for hours."

Participants were also invited to write in coping strategies. These results included, "friends," "family," "humor,"

Table 5 Correlation of burnout, compassion satisfaction, and secondary stress

\begin{tabular}{lcc}
\hline & Burnout & $\begin{array}{l}\text { Compassion } \\
\text { satisfaction }\end{array}$ \\
\hline Burnout & & \\
Compassion satisfaction & $-0.74^{*}$ & \\
Secondary stress & $+0.53^{*}$ & $-0.33^{*}$ \\
\hline
\end{tabular}

$* p<.0001$ "taking joy in accomplishments," "tough it out," "cry," "Netflix," "teaching," "SNRI's," "wine with dinner," and "not very well."

\section{Discussion}

This project reveals several intriguing associations between burnout, secondary stress, and compassion satisfaction, which are tightly correlated (Table 5). There is a constellation of emotional coping strategies and practices that suggests a disposition of wellness and tenacity among those physicians with lower levels of burnout, secondary stress, and higher levels of compassion satisfaction. These physicians have higher levels of positive copings strategies such as grit, acceptance, active coping, positive reframing, strategy planning, and even physical exercise. These findings have very provocative implications for the emotional health of the physician workforce. Can positive emotional coping be taught? Many studies show training in resiliency strategies reduce burnout, perceived stress, and improve perceived ability to manage stress but there are few studies among physicians (Robertson et al., 2015). Of note, a pilot study of an 8-week, abbreviated mindfulness-based stress reduction class designed for primary care physicians showed reduction in Maslach burnout subscales, but no improvement in resilience (Fortney, Luchterhand, Zakletskaia, Zgierska, \& Rakel, 2013). Another study by West et al (2014) organized bi-monthly small-group training sessions for physicians to learn mindfulness and coping skills for 9 months. This program demonstrated improved engagement, empowerment, and decreased depersonalization among the intervention group. These effects were maintained at a 12-month follow-up. Providing opportunities to optimize resilience and improve emotional coping strategies can be an important resource for physician wellness (West et al., 2014). Institutional leadership would benefit from formal collaboration with psychologists, and other mental health providers to design, implement, and evaluation interventions to address burnout.

In this study, there are two extrinsic, psychosocial variables that are associated with lower burnout, secondary stress, and compassion satisfaction: institutional support and friendship. Friendship has been scarcely studied in the literature. Physicians who endorsed stronger friendships reported lower burnout and greater compassion satisfaction. Similarly, those physicians who felt well supported by their institution also had lower burnout and greater compassion satisfaction. While causation cannot be implied, social support, friendships, and institutional culture may play an important role to mitigate burnout. Social support has been associated with job satisfaction in several studies among nurses but has not been well studied among physicians (Woodhead, 
Northrop, \& Edelstein, 2016; Orgambídez-Ramos \& de Almeida, 2017).

In this study, religion and spirituality had no correlation with any burnout domain. Neither religious attendance, internal practices, nor communal practices were associated with any ProQol domains. Religious involvement has been shown in many studies to correlate with improved mental health, especially among those with depression, anxiety, and suicidality (Koenig \& Bussing, 2010). Further study is needed to explore this important resource among physicians.

Linzer et al (2014) suggests several steps that organizations should employ to optimize physician wellness. These include making wellness a measurable quality indicator, similar to catheter-related infections and hospital readmission rates, which promotes tracking and dialogue around workplace culture. Other evidence-based interventions include mindfulness training, optimizing the electronic medical record, and improving resources in the work environment such as job-sharing and increased ancillary staff (Linzer et al., 2014). In addition, institutions need to hire leaders who foster a collaborative, supportive environment. Physician retention and satisfaction has an important impact upon institutions. The cost of replacing a primary care physician is 2-3 times a physician salary (Buchbinder, Wilson, Melick, \& Powe, 1999; Misra-Hebert, Kay, \& Stoller, 2004). Physicians who experience burnout are more likely to commit medical errors (Shanafelt et al., 2010; Williams et al., 2007). In an era when insurance companies withhold payments and penalize hospitals for readmission rates and poor quality indicators, addressing physician satisfaction can potentially save lives and positively confer cost savings.

Limitations to this project should be considered. First, the response rate of $33 \%$ is low, but similar to cohort studies of this type (Lavanchy et al., 2004; Vicentic et al., 2013). While adequately powered, the burnout prevalence of those who did not respond to the questionnaire is unknown. Yet, the prevalence of burnout was consistent with prior studies of physicians (Shanafelt et al., 2019). Second, these associations are observational. It is difficult to infer causation. For example, does greater resilience prevent secondary stress? Or, does the secondary stress exhaust the resilience of the physician? Further, as this was a convenience sample, we do not appreciate how physician burnout fluctuates over time. Is burnout a stable personality type or does burnout fluctuates due to the stresses of the job?

Many survey-type studies have been performed exploring these issues. A qualitative study among satisfied physicians exploring positive emotional coping strategies, workplace environment, and social networks would be an important contribution to the literature. This would include potentially supportive relationships such as marriage satisfaction, family life, or community involvement. Also, the nature of institutional support is not well-defined in the literature and merits further definition. Institutional support could imply a collegial environment, a corps d'esprit, that sustains physicians amidst work stress. Institutional support could also imply job-related operational factors such as job-sharing, on-site child-care, or ready access to mental health services. In addition to a qualitative study, longitudinal intervention trials of wellness programs, such as resilience and mindfulness training, would inform gaps in the literature.

\section{Conclusion}

Physician wellness is an important element of the healthcare system that has implications for patient safety and quality care. Optimizing physician wellness through institutional efforts to enhance work-life balance, job flexibility, and individual emotional coping is important for the future of patient care.

Acknowledgements The author wishes to thank Dr. Robert Nardino and Dr. Ivelisse Verrico for their support with data collection and conception of this project.

\section{Compliance with Ethical Standards}

Conflict of interest Benjamin R. Doolittle has no conflict of interest.

Human and Animal Rights and Informed Consent The Yale University School of Medicine Human Investigation Committee approved the study protocol after expedited review. Leadership from the American College of Physicians gave permission for the survey to be emailed to membership.

\section{References}

Bodenheimer, T., \& Sinsky, C. (2014). From triple to quadruple aim: Care of the patient requires care of the provider. The Annals of Family Medicine, 12, 573-576. https://doi.org/10.1370/afm.1713.

Bonelli, R. M., \& Koenig, H. G. (2013). Mental disorders, religion and spirituality 1990 to 2010: A systematic evidence-based review. Journal of Religion and Health, 52, 657-673. https://doi. org/10.1007/s10943-013-9691-4.

Buchbinder, S. B., Wilson, M., Melick, C. F., \& Powe, N. R. (1999). Estimates of costs of primary care physician turnover. The American Journal of Managed Care, 5, 1431-1438.

Carver, C. S., Scheier, M. F., \& Weintraub, J. K. (1989). Assessing coping strategies: A theoretically based approach. Journal of Personality and Social Psychology, 56(2), 267. https://doi. org/10.1037/0022-3514.56.2.267.

Chopra, S. S., Sotile, W. M., \& Sotile, M. O. (2004). Physician burnout. JAMA, 291(5), 633-633. https://doi.org/10.1001/jama.291.5.633.

Doolittle, B. R., Windish, D. M., \& Seelig, C. B. (2013). Burnout, coping, and spirituality among internal medicine resident physicians. Journal of Graduate Medical Education, 5, 257-261. https://doi. org/10.4300/jgme-d-12-00136.1.

Duckworth, A. L., Peterson, C., Matthews, M. D., \& Kelly, D. R. (2007). Grit: Perseverance and passion for long-term goals. 
Journal of Personality and Social Psychology, 92, 1087-1101. https://doi.org/10.1037/0022-3514.92.6.1087.

El-bar, N., Levy, A., Wald, H. S., \& Biderman, A. (2013). Compassion fatigue, burnout and compassion satisfaction among family physicians in the Negev area: A cross-sectional study. Israel Journal of Health Policy Research, 2, 31-39. https://doi. org/10.1186/2045-4015-2-31.

Fortney, L., Luchterhand, C., Zakletskaia, L., Zgierska, A., \& Rakel, D. (2013). Abbreviated mindfulness intervention for job satisfaction, quality of life, and compassion in primary care clinicians: A pilot study. The Annals of Family Medicine, 11, 412-420. https://doi. org/10.1370/afm.1511.

Halliday, L., Walker, A., Vig, S., Hines, J., \& Brecknell, J. (2017). Grit and burnout in UK doctors: A cross-sectional study across specialties and stages of training. Postgraduate Medical Journal, 93, 389-394. https://doi.org/10.1136/postgradmedj-2015-133919.

Koenig, H. G., \& Büssing, A. (2010). The Duke University Religion Index (DUREL): A five-item measure for use in epidemiological studies. Religions, 1, 78-85. https://doi.org/10.3390/rel1010078.

Lavanchy, M., Connelly, I., Grzybowski, S., Michalos, A. C., Berkowitz, J., \& Thommasen, H. V. (2004). Determinants of rural physicians' life and job satisfaction. Social Indicators Research, 69, 93-101. https://doi.org/10.1023/b:soci.0000032662.79752.e8.

Linzer, M., Levine, R., Meltzer, D., Poplau, S., Warde, C., \& West, C. P. (2014). 10 bold steps to prevent burnout in general internal medicine. Journal of General Internal Medicine, 29, 18-20. https ://doi.org/10.1007/s11606-013-2597-8.

Maslach, C., Jackson, S. E., \& Leiter, M. P. (1996). Maslach Burnout Inventory Manual (3rd ed.). Palo Alto, CA: Consulting Psychologists Press.

Misra-Hebert, A. D., Kay, R., \& Stoller, J. K. (2004). A review of physician turnover: rates, causes, and consequences. American Journal of Medical Quality, 19, 56-66. https://doi.org/10.1177/10628 6060401900203.

Orgambídez-Ramos, A., \& de Almeida, H. (2017). Work engagement, social support, and job satisfaction in Portuguese nursing staff: A winning combination. Applied Nursing Research, 36, 37-41. https ://doi.org/10.1016/j.apnr.2017.05.012.

Robertson, I. T., Cooper, C. L., Sarkar, M., \& Curran, T. (2015). Resilience training in the workplace from 2003 to 2014: A systematic review. Journal of Occupational and Organizational Psychology, 88, 533-562. https://doi.org/10.1111/joop.12120.

Sedgwick, P. (2012). Multiple significance tests: The Bonferroni correction. BMJ, 344, e509. https://doi.org/10.1136/bmj.e509.

Shanafelt, T. D., Balch, C. M., Bechamps, G., Russell, T., Dyrbye, L., Satele, D., et al. (2010). Burnout and medical errors among American surgeons. Annals of Surgery, 251, 995-1000. https:// doi.org/10.1097/sla.0b013e3181bfdab3.

Shanafelt, T. D., Dyrbye, L. N., Sinsky, C., Hasan, O., Satele, D., Sloan, J., et al. (2016). Relationship between clerical burden and characteristics of the electronic environment with physician burnout and professional satisfaction. Mayo Clinic Proceedings, 91, 836-848. https://doi.org/10.1016/j.mayocp.2016.05.007.

Shanafelt, T. D., West, C. P., Sinsky, C., Trockel, M., Tutty, M., Satele, D. V., ... Dyrbye, L. N. (2019). Changes in burnout and satisfaction with work-life integration in physicians and the general US working population between 2011 and 2017. Mayo Clinic Proceedings, 94, 1681-1694. https://doi.org/10.1016/j.mayoc p.2018.10.023.

Stamm, B. H. (2005). The ProQol manual. Brooklandville, MD: Sidran Press.

Topolski, T. D., LoGerfo, J., Patrick, D. L., Williams, B., Walwick, J., \& Patrick, M. M. B. (2006). Peer reviewed: The Rapid Assessment of Physical Activity (RAPA) among older adults. Preventing Chronic Disease, 3, a118.

Vicentic, S., Gasic, M. J., Milovanovic, A., Tosevski, D. L., Nenadovic, M., Damjanovic, A., ... Jovanovic, A. A. (2013). Burnout, quality of life and emotional profile in general practitioners and psychiatrists. Work, 45, 129-138. https://doi.org/10.3233/wor-121484.

Woodhead, E. L., Northrop, L., \& Edelstein, B. (2016). Stress, social support, and burnout among long-term care nursing staff. Journal of applied gerontology, 35, 84-105. https://doi.org/10.1177/07334 64814542465.

West, C. P., Dyrbye, L. N., Erwin, P. J., \& Shanafelt, T. D. (2016). Interventions to prevent and reduce physician burnout: A systematic review and meta-analysis. The Lancet, 388, 2272-2281. https ://doi.org/10.1016/s0140-6736(16)31279-x.

West, C. P., Dyrbye, L. N., Rabatin, J. T., Call, T. G., Davidson, J. H., Multari, A., ... Shanafelt, T. D. (2014). Intervention to promote physician well-being, job satisfaction, and professionalism: A randomized clinical trial. JAMA Internal Medicine, 174, 527-533. https://doi.org/10.1001/jamainternmed.2013.14387.

Williams, E. S., Manwell, L. B., Konrad, T. R., \& Linzer, M. (2007). The relationship of organizational culture, stress, satisfaction, and burnout with physician-reported error and suboptimal patient care: Results from the MEMO study. Health Care Management Review, 32, 203-212. https://doi.org/10.1097/01.hmr.0000281626 .28363 .59 .

Yost, W. B., Eshelman, A., Raoufi, M., \& Abouljoud, M. S. (2005). A national study of burnout among American transplant surgeons. Transplantation Proceedings, 37, 1399-1401. https://doi. org/10.1016/j.transproceed.2005.01.055.

Publisher's Note Springer Nature remains neutral with regard to jurisdictional claims in published maps and institutional affiliations. 